order: participants, materials, procedures, setting and intervention).

Conclusions This rehabilitation case-study shows problems of clinical replicability of RCTs on complex interventions and suggests the need to better define some clinical items not described by classical methodological checklists like CONSORT.

\section{CONTINUOUS OUTCOME MEASURES - CONUNDRUMS AND CONVERSIONS CONTRIBUTING TO CLINICAL APPLICATION OBJECTIVES}

1,2Martin Mayer. ${ }^{1}$ EBSCO Health, Ipswich, MA, USA; ${ }^{2}$ Cone Health, Greensboro, NC, USA

10.1136/bmjebm-2019-EBMLive.13

Objectives To discuss how continuous outcome measures are commonly used to measure patient-relevant outcomes, the obstacles in employing these measures clinically, and methods available to facilitate use of bodies of evidence comprised of continuous outcomes.

Method Many patient-relevant outcomes, particularly quality of life measures such as pain or function, are routinely measured on a continuous scale. However, the interpretation of continuous outcomes can be difficult, particularly when considering application to clinical practice and shared decisionmaking. Making matters worse is the frequent existence of multiple scales for any given construct. Therefore, quantitative syntheses of literature must find a way to combine different scales into a 'common language', and the longest-standing and most frequently used method to do so is the standardized mean difference. Unfortunately, the standardized mean difference is even more difficult to interpret clinically. However, there are validated methods to make these measures easier to understand and apply clinically. This presentation explores these issues and offers a resource to help make these continuous measures more clinically useful.

Results Different methods to amplify the clinical use of continuous outcome measures have been discussed for at least three decades now, some better known than others. These methods seek to estimate the proportion of patients expected to achieve a specified degree of benefit or harm based on the observed continuous outcome. Exploring the strengths, limitations, and judicious use of these methods facilitates a greater understanding of how continuous outcomes data might be usable for clinical and shared decision-making. Of the methods that have been validated for this purpose, approximated and observed estimates of the proportion of people who achieved a specified degree of change in the continuous outcome measure have shown considerable consistency.

Conclusions Although not necessarily a panacea, methods facilitating conversion of continuous outcomes data to proportions of people expected to achieve a specified degree of benefit adds considerably to our ability to make sense and clinical use of continuous outcome measures. As such, when these methods are used appropriately, they add substantially to our evidence-based medicine and shared decision-making 'toolkit'.

\section{VACCINE INJURY REDRESS PROGRAMMES: A SYSTEMATIC INTEGRATIVE REVIEW}

Martin Keane, Tonya Moloney, Caitriona Lee, Michael O'Sullivan, Jean Long. Health Research Board, Dublin, Ireland

\subsection{6/bmjebm-2019-EBMLive.14}

Objectives This review investigated the design features of vaccine injury redress programmes that are thought to impact on the operating costs, timely access to compensation, number of applicants, and the volume and costs of awards. In addition, we ascertained if such programmes enjoy public acceptance. Vaccine injury redress programmes are available in 20 jurisdictions; only 11 jurisdictions have completed detailed papers on their set up and operation, namely, China, Denmark, Finland, Japan, Korea, Norway, New Zealand, Sweden, Taiwan, UK, and USA.

Method We chose the Integrative Review approach to synthesise an array of theoretical and empirical data to answer our questions. We searched in MEDLINE, CINAHL, Scopus, Web of Science, Cochrane Library, HeinOnline and LegalTrac. We identified 2,838 papers and selected 33 papers that provided relevant data to answer at least one of our questions. We developed a conceptual schema based on key design features of vaccine injury redress programmes to code and extract data. The design features identified were: approach, administrator, funding source, vaccines covered, injuries covered, claims and decisionmaking process, standard of proof, litigation rights, costs awarded, and cost controls. We analysed the data using the constant comparative method as none of the papers included in our review asked either the same or similar questions to ours.

Results The treatment injury compensation scheme in New Zealand and the drug injury scheme in the four Nordic countries have improved access to compensation by removing the concept of negligence and lowering the standard of proof required. Removing negligence has also reduced administrative and legal costs in the five countries. Public awareness of and support for the scheme is high in New Zealand and it has buy-in from physicians. The schemes in these five countries are aligned to national employment insurance schemes and publicly funded health care.

The USA, UK and four countries in Asia operate standalone vaccine injury redress programmes. The USA, UK and China employs a strict standard of proof which reduces timely access to redress and keeps the approval rate for claims low. Korea and Taiwan operate a more relaxed standard of proof, and Taiwan resolves claims in a timely fashion. In Japan, higher compensation is awarded to claimants judged to have protected 'the herd immunity'. 